\title{
Max-Consensus in a Max-Plus Algebraic Setting: The Case of Switching Communication Topologies
}

\author{
Behrang Monajemi Nejad* Sid Ahmed Attia* \\ Jörg Raisch ${ }^{*, * *}$ \\ * Fachgebiet Regelungssysteme, Technische Universität Berlin, Berlin, \\ Germany(e-mail:nejad@control.tu-berlin.de,attia@ieee.org, \\ raisch@control.tu-berlin.de). \\ ** Systems and Control Theory Group, Max-Planck-Institut für \\ Dynamik komplexer technischer Systeme, Magdeburg, Germany
}

\begin{abstract}
Because of their use for distributed decision making, consensus algorithms have attracted a lot of interest in recent years. Coordination between entities requires that they share information over a network, and develop a consistent view regarding objectives and relevant information on the environment, i.e., reach a consensus. In practice, communication topologies may change over time, either as a consequence of disturbances or in an attempt to improve performance. Max-consensus is a specific consensus algorithm, which is particularly important in applications such as minimum time rendezvous and leader election. In this contribution, we propose an approach to analyze max-consensus algorithms in time-variant communication topologies, which is based on max-plus algebra. In this framework max-consensus algorithms become piecewise linear and may be analyzed easily. The conditions needed to achieve maxconsensus and the convergence rate of the algorithm for different communication graphs are studied. This contribution is an extension of the work in Monajemi Nejad et al. (2009), where max-consensus was studied for time-invariant communication topologies.
\end{abstract}

Keywords: Consensus Algorithms, Max-Plus Algebra, Switched Max-Plus Linear Systems, Networked Control Systems, Cooperative Control

\section{INTRODUCTION}

Cooperation in a network occurs if its elements share information over the (possibly time varying) network topology, and develop a consistent view regarding objectives and relevant information on the environment. To realize this, two ingredients are necessary. The information state is a network member's local estimate of the relevant variables, and the consensus algorithm describes how these local estimates change through information exchange between network members. A consensus is said to be reached if all local information states coincide (Ren and Beard (2008)).

Various applications of distributed cooperative systems have been reported in the literature (e.g., Ren and Beard (2008), Roy et al. (2005), Wang et al. (2004), Gil et al. (2008) and Feddema et al. (2002)). In some applications, such as minimum-time rendezvous or leader election (Lynch (1996)), a special class of consensus algorithms called max-consensus has to be used. In this case a consensus is said to be achieved, if all the members in the network have the same information state, which is required to be the maximum of all initial information states.

The differential or difference equations describing maxconsensus algorithms are in general time-variant, since communication topologies, modeled by directed graphs, are often dynamic. For example, communication links among nodes might be unreliable due to disturbances such as imperfect sensors, communication dropout, and noisy and unreliable communication links. For effective cooperation, a team working in a network must be able to respond to unanticipated situations or changes occurring in the environment. Another important reason to analyze maxconsensus in dynamic communication topologies is the possibility to optimize the communication performance, e.g., with respect to energy consumption or convergence rate. Max-consensus in a continuous time framework and for the case of fixed communication topologies has been recently proposed and studied in Cortés (2008) and TahbazSalehi and Jadbabaie (2006). Convergence conditions for a number of consensus problems have been studied for various dynamic communication topologies (see, e.g. OlfatiSaber and Murray (2004), Cao et al. (2008) and Moreau (2005)).

In this contribution, we propose an approach to analyze max-consensus algorithms which is based on max-plus algebra (e.g., Baccelli et al. (2001), Heidergott et al. (2005) and De Schutter and van den Boom (2008)). In this framework max-consensus algorithms become linear (for constant communication topologies) and can be represented as a switched linear system (for time-varying communication toplogies). In particular, we investigate conditions under which max-consensus algorithms converge under switching in communication topologies. 
The paper is organized as follows: Section 2 summarizes the necessary elements from graph theory and max-plus algebra. Section 3 introduces the basic concepts of maxconsensus in a max-plus algebraic setting. Section 4 provides convergence conditions for so-called strong maxconsensus in switching topologies. Section 5 does the same for so-called weak max-consensus. Finally some simulation results and a conclusion are given in Section 6 and Section 7 .

\section{GRAPH THEORY AND MAX-PLUS ALGEBRA}

In this section we collect a few basic facts on graphs and max-plus algebra that are needed in the sequel.

\subsection{Graph Theory}

Information exchange between nodes in a network is modeled by means of directed or undirected graphs. A directed graph $\mathcal{G}$ is a pair $(\mathcal{N}, \mathcal{E})$, where $\mathcal{N}=\{1, \cdots, n\}$ is a finite nonempty node set and $\mathcal{E} \subseteq \mathcal{N} \times \mathcal{N}$ is a set of ordered pairs of nodes, called edges. Additionally, in weighted graphs each edge is equipped with a weight. An edge may connect a node to itself (self loop). Existence of an edge $(j, i) \in \mathcal{E}$ denotes that node $i$ can obtain information from node $j$. If $(j, i)$ is an edge in a directed graph, node $j$ is called a predecessor of node $i$. $\mathcal{J}_{i}$ is the set of predecessor nodes of node $i$, i.e., $\mathcal{J}_{i}=\{j \in \mathcal{N} \mid(j, i) \in \mathcal{E}\}$. A path is a sequence of nodes $\left(i_{1}, \cdots, i_{p}\right), p>1$, such that $i_{j}$ is a predecessor of $i_{j+1}, j=1, \cdots, p-1$. An elementary path is a path in which no node appears more than once. A directed graph is said to be strongly connected if there is a path from any node to any other node in the graph, and it is called weakly connected if the graph obtained by adding an edge $(i, j)$ for every existing edge $(j, i)$ in the original graph is strongly connected. A rooted directed tree is a directed graph in which every node has exactly one predecessor node, called parent, except for one node, called the root, which has no parent and from which there is a directed path to every other node. $\left(\mathcal{N}_{1}, \mathcal{E}_{1}\right)$ is a subgraph of $(\mathcal{N}, \mathcal{E})$, if $\mathcal{N}_{1} \subseteq \mathcal{N}$ and $\mathcal{E}_{1}$ is a set of edges $(j, i)$ from $\mathcal{E}$, such that $i, j \in \mathcal{N}_{1}$.

The union graph $\bigcup_{i} \mathcal{G}_{i}$ of the directed graphs $\mathcal{G}_{i}\left(\mathcal{N}, \mathcal{E}_{i}\right)$ is the graph $\left(\mathcal{N}, \mathcal{E}_{\cup}\right)^{\imath}$, where

$$
\mathcal{E}_{\cup}:=\bigcup_{i} \mathcal{E}_{i}
$$

The intersection graph $\bigcap \mathcal{G}_{i}$ of the directed graphs $\mathcal{G}_{i}$ $\left(\mathcal{N}, \mathcal{E}_{i}\right)$ is the graph $\left(\mathcal{N}, \mathcal{E}_{\cap}^{i}\right)$, where

$$
\mathcal{E}_{\cap}:=\bigcap_{i} \mathcal{E}_{i}
$$

More details on graph theory can be found in, e.g., Diestel (2005), Baccelli et al. (2001).

\subsection{Max-Plus Algebra}

Max-plus algebra, e.g., Baccelli et al. (2001), Heidergott et al. (2005) and De Schutter and van den Boom (2008), represents a powerful tool for simulation and analysis of a class of timed cyclic discrete-event systems and allows for a compact representation of weighted graphs.

Max-plus algebra consists of two binary operations, $\oplus$ and $\otimes$, on the set $\mathbb{R}_{\max }:=\mathbb{R} \cup\{-\infty\}$. The operations are defined as follows:

$$
\begin{aligned}
& a \oplus b:=\max (a, b), \\
& a \otimes b:=a+b .
\end{aligned}
$$

The neutral element of max-plus addition $\oplus$ is $-\infty$, denoted as $\varepsilon$. The neutral element of multiplication $\otimes$ is 0 , denoted as e. The elements $\varepsilon$ and e are also referred to as the zero and one element of max-plus algebra. Similar to conventional algebra, associativity, commutativity, and distributivity of multiplication over addition also hold for the max-plus algebra. Both operations can be extended to matrices in a straightforward way. For $A, B \in \mathbb{R}_{\max }^{m \times n}$,

$$
(A \oplus B)_{i j}:=a_{i j} \oplus b_{i j}, \quad i=1, \cdots, m, j=1, \cdots, n .
$$

For $A \in \mathbb{R}_{\max }^{m \times n}, B \in \mathbb{R}_{\max }^{n \times q}$,

$$
\begin{array}{r}
(A \otimes B)_{i j}:=\bigoplus_{k=1}^{n}\left(a_{i k} \otimes b_{k j}\right)=\max _{k}\left(a_{i k}+b_{k j}\right), \\
i=1, \cdots, m, j=1, \cdots, q .
\end{array}
$$

Note that, as in conventional algebra, the multiplication symbol $\otimes$ is often omitted.

In the sequel, we also need matrices of zero elements, denoted by $N$, and of one elements, denoted by $E$. The identity matrix $I$ is a square matrix with

$$
(I)_{i j}:= \begin{cases}\mathrm{e} & \text { for } i=j \\ \varepsilon & \text { else. }\end{cases}
$$

For any matrix $A \in \mathbb{R}_{\max }^{n \times n}$, its precedence graph $\mathcal{G}(A)$ is defined in the following way: it has $n$ nodes, denoted by $1, \cdots, n$, and $(j, i)$ is an edge if and only if $a_{i j} \neq \varepsilon$. In this case $a_{i j}$ is the weight of edge $(j, i)$. Then

- A path in $\mathcal{G}(A)$ is a sequence of $p>1$ nodes, denoted by $\rho:=i_{1}, \ldots, i_{p}$, such that $a_{i_{k+1} i_{k}} \neq \varepsilon, k=$ $1, \ldots, p-1$.

- The weight of a path $\rho$ denoted by $|\rho|_{w}$ is defined as $|\rho|_{w}:=\sum_{k=1}^{p-1} a_{i_{k+1} i_{k}}$ and its corresponding length as $|\rho|_{l}:=p-1$.

- The length of the shortest path from node $i$ to node $j$ is denoted by $|i, j|_{\text {min }}$.

- $\left(A^{k}\right)_{i j}$ represents the maximal weight of all paths of length $k$ from node $j$ to node $i$, where

$$
A^{k}:=\underbrace{A \otimes A \otimes \ldots \otimes A}_{(k-1) \text {-times multiplication }} \quad, \quad k \geq 1
$$

and $A^{0}=I$.

\section{PROBLEM SETUP}

The following example from Monajemi Nejad et al. (2009) illustrates the concept of max-consensus. A group of agents is assigned to meet as early as possible at a particular place. Each agent needs a certain minimum time to get to the meeting place. This means that the only possibility 
to achieve an agreement on the minimal feasible meeting time is to take the largest value of all the agents' minimum times. A possible centralized solution to this problem could be a simultaneous communication between all agents in the group, to arrange a time when the group will meet. For this purpose the time of this communication should be known to the group. Thus, the centralized approach does not help to solve the problem. A distributed approach to the problem would be for each agent to communicate, one at a time, with a subset of the group. An update of his current estimate of the meeting time may be performed by taking the maximum of this estimate and that of the contacted agents. The question to be answerded in this paper is: under which conditions does this strategy guarantee that all group members' estimates regarding the meeting time will converge? For the case that the communication topology is fixed, the conditions and results are given in Monajemi Nejad et al. (2009). In the following, we investigate the case where the communication topology may vary over time.

Note that time-varying communication topologies also cover the case of asynchronous communication, where the information exchange between neighbors is determined by individual graphs. Furthermore, we consider the discretetime case, where communication instants may either be defined by a clock or by the occurrence of external events.

The scenario described above can be formally stated as follows:

- The set of graph members is denoted by $\mathcal{N}=$ $\{1, \cdots, n\}$.

- Information between graph members can be exchanged at discrete instants of time, $t_{k}, k=0,1,2, \cdots$.

- The communication topology at time $t_{k}$ is modeled by a directed graph $\mathcal{G}_{k}=\left(\mathcal{N}, \mathcal{E}_{k}\right)$, where $(j, i) \in$ $\mathcal{E}_{k}, j, i \in \mathcal{N}$, means that node (graph member) $i$ receives information from node (graph member) $j$. The weights of all edges are equal to e. Furthermore, $(i, i) \in \mathcal{E}_{k} \forall i \in \mathcal{N}$.

- As $n$ is finite, and all edges have weight e, there only exists a finite number of possible communication graphs, i.e. $\mathcal{G}_{k} \in\left\{\mathcal{G}_{(1)}, \cdots, \mathcal{G}_{\left(n_{m}\right)}\right\} \forall k$.

- The information state of agent $i$ at time $t_{k}$ is denoted by $x_{i}(k)$ and it is updated via the max-consensus algorithm

$$
x_{i}(k+1)=\max _{j \in \mathcal{J}_{i, k}}\left\{x_{j}(k)\right\}, \quad i=1 \cdots n,
$$

where $\mathcal{J}_{i, k}$ is the set of predecessor nodes of node $i$ in the graph $\mathcal{G}_{k}$, i.e. $J_{i, k}:=\left\{j \mid(j, i) \in \mathcal{E}_{k}\right\}$.

Definition 3.1. (Max-Consensus). For a given sequence of graphs, $\mathcal{G}_{k}, k=0,1, \cdots$, a vector of initial states $x_{0}:=$ $\left(x_{1}(0), \cdots, x_{n}(0)\right)^{T} \in \mathbb{R}^{n}$, and the consensus algorithm (5), max-consensus is said to be achieved, if $\exists K \in \mathbb{N}_{0}$ such that

$$
\begin{aligned}
x_{i}(k) & =x_{j}(k) \\
& =\max \left\{x_{1}(0), \cdots, x_{n}(0)\right\}, \quad \forall k \geq K, \quad \forall i, j \in \mathcal{N} .
\end{aligned}
$$

If (6) holds for all $x_{0} \in \mathbb{R}^{n}$, strong max-consensus is achieved. If (6) only holds for a subset of initial states, $X_{0} \subseteq \mathbb{R}^{n}$, weak max-consensus is achieved.
Clearly, strong max-consensus is a property of the sequence of graphs $\mathcal{G}_{k}, k=0,1, \cdots$ only, whereas weak maxconsensus depends on the graph sequence and $x_{0}$.

In a max-plus framework, (5) becomes a switched linear system. To see this, we associate a matrix $A_{(l)}$ to each graph $\mathcal{G}_{(l)}, l \in\left\{1, \cdots, n_{m}\right\}$, such that $\mathcal{G}_{(l)}$ is the precedence graph of $A_{(l)}$. We can then write (5) as

$$
x_{i}(k+1)=\bigoplus_{j \in \mathcal{J}_{i, k}}\left(x_{j}(k)\right), \quad i=1, \cdots, n,
$$

or, equivalently,

$$
x(k+1)=A_{k} \otimes x(k),
$$

where $x(k)=\left(x_{1}(k), \cdots, x_{n}(k)\right)^{T}$ is the vector of information states and $A_{k} \in\left\{A_{(1)}, \cdots, A_{\left(n_{m}\right)}\right\}$ is a matrix in $\mathbb{R}_{\max }^{n \times n}$ associated with the graph $\mathcal{G}_{k}$.

Recursive application of (8) to $x_{0}$ provides

$$
x(k)=A_{k-1} \otimes A_{k-2} \otimes \cdots \otimes A_{0} \otimes x_{0} .
$$

We will investigate conditions which

- guarantee strong (weak) max-consensus for a specific sequence of graphs $\mathcal{G}_{k}$, or, correspondingly, for a given sequence of matrices $A_{k}, k=0,1,2, \cdots$.

- guarantee strong (weak) max-consensus for an arbitrary sequence $\mathcal{G}_{k}$ or $A_{k}$, with $A_{k} \in\left\{A_{(1)}, \cdots, A_{\left(n_{m}\right)}\right\}$, $k=0,1,2, \cdots$.

Before we do this, let us briefly summarize results from Monajemi Nejad et al. (2009) for the case of a fixed communication topology. There, the sequence $\mathcal{G}_{k} k=$ $0,1, \cdots$ reduces to a single (constant) graph $\mathcal{G}=(\mathcal{N}, \mathcal{E})$. In Monajemi Nejad et al. (2009), we have shown, that in this case strong max-consensus is achieved if and only if $\mathcal{G}$ is strongly connected. Then, the required number of communication instants is the maximum of the shortest path lengths between any pair of nodes in $\mathcal{G}$, i.e., $n-1$ in the worst case. Weak max-consensus is achieved if and only if there exist rooted directed trees in $\mathcal{G}$ which span $\mathcal{N} \backslash \mathcal{N}_{1}$ and have roots in $\mathcal{N}_{1}$, where $\mathcal{N}_{1} \subseteq \mathcal{N}$ is the set of nodes with maximal initial information state. The required number of communication instants to achieve consensus is then

$$
\max _{i \in \mathcal{N} \backslash \mathcal{N}_{1}}\left(\min _{j \in \mathcal{N}_{1}}\left\{|j, i|_{\min }\right\}\right)
$$

\section{STRONG MAX-CONSENSUS IN SWITCHING TOPOLOGIES}

Let $\mathcal{S}$ be the set of all sequences of matrices from the set $\mathcal{A}=\left\{A_{(1)}, \cdots, A_{\left(n_{m}\right)}\right\}$. Recall that $A_{(l)} \in \mathbb{R}_{\max }^{n \times n}$, such that $\mathcal{G}_{(l)}=\left(\mathcal{N}, \mathcal{E}_{(l)}\right)$ is the precedence graph of $A_{(l)}$, $l=1, \cdots, n_{m}$. This implies that all diagonal elements of $A_{(l)}$ are equal to e, and that all off-diagonal elements are either $\varepsilon$ or e (as $(i, i) \in \mathcal{E}_{(l)} \forall i \in \mathcal{N}$ and all edges have weight e). 


\subsection{Strong Max-Consensus in Switching Topologies for Specific Switching Sequences}

Theorem 4.1. Let $A_{k}, k=0,1,2, \cdots$, be a sequence of matrices with $A_{k}=A_{\left(l_{k}\right)}$. A necessary and sufficient condition for strong max-consensus is that

$$
A_{\left(l_{k-1}\right)} \otimes A_{\left(l_{k-2}\right)} \otimes \cdots \otimes A_{\left(l_{1}\right)} \otimes A_{\left(l_{0}\right)}=E,
$$

for some $k \in \mathbb{N}$.

Proof 1. (10) implies $x(k)=E \otimes x_{0}$, i.e.,

$$
\begin{aligned}
x_{i}(k) & =\bigoplus_{j=1, \cdots, n}\left(x_{j}(0)\right) \\
& =\max \left\{x_{1}(0), \cdots, x_{n}(0)\right\}, \quad i=1, \cdots, n .
\end{aligned}
$$

Furthermore, because of the special form of the matrices $A_{(l)},(10)$ implies that $\forall q>k$

hence

$$
A_{\left(l_{q-1}\right)} \otimes \cdots \otimes A_{\left(l_{k-1}\right)} \otimes \cdots \otimes A_{\left(l_{0}\right)}=E
$$

$$
x_{i}(q)=\max \left\{x_{1}(0), \cdots, x_{n}(0)\right\}, \quad i=1, \cdots, n .
$$

Necessity can be shown as follows: if $A_{\left(l_{k-1}\right)} \otimes A_{\left(l_{k-2}\right)} \otimes$ $\cdots \otimes A_{\left(l_{1}\right)} \otimes A_{\left(l_{0}\right)} \neq E \forall k$, then $(\forall k) \exists p, q \in \mathcal{N}$ s.t. $\left(A_{\left(l_{k-1}\right)} \otimes A_{\left(l_{k-2}\right)} \otimes \cdots \otimes A_{\left(l_{1}\right)} \otimes A_{\left(l_{0}\right)}\right)_{p q}=\varepsilon$, i.e., $x_{p}(k)$ does not depend on $x_{q}(0)$. Hence, if $x_{q}(0)$ is the maximum element of $x_{0}$, max-consensus is not achieved.

In the following, we will also need a number of technical results.

Lemma 4.1. Let $\mathcal{G}^{\prime}=\left(\mathcal{N}^{\prime}, \mathcal{E}^{\prime}\right)$ be a subgraph of a strongly connected graph $\mathcal{G}=(\mathcal{N}, \mathcal{E})$, with $\mathcal{N}^{\prime} \subset \mathcal{N}$. Then there exists at least one edge $(i, j) \in \mathcal{E}$ with $i \in \mathcal{N}^{\prime}$ and $j \in \mathcal{N} \backslash$ $\mathcal{N}^{\prime}$.

Proof 2. The proof can be easily shown by contradiction.

Lemma 4.2. Consider the matrices $A, B \in \mathbb{R}_{\max }^{n \times n}$ and the corresponding precedence graphs $\mathcal{G}_{A}=\left(\mathcal{N}, \mathcal{E}_{A}\right)$ and $\mathcal{G}_{B}=\left(\mathcal{N}, \mathcal{E}_{B}\right)$. Let $(i, i) \in \mathcal{E}_{A}$ and $(i, i) \in \mathcal{E}_{B} \forall i \in \mathcal{N}$, and let all edges have weight e, i.e., the diagonal elements of $A$ and $B$ are all equal to e, while the off-diagonal elements are either e or $\varepsilon$. Then, all edges of $\mathcal{G}_{A}$ and $\mathcal{G}_{B}$ are also edges of the precedence graph $\mathcal{G}_{A \otimes B}=\left(\mathcal{N}, \mathcal{E}_{A \otimes B}\right)$ of the matrix $A \otimes B$, i.e.

$$
\mathcal{E}_{\mathcal{A} \otimes \mathcal{B}} \supseteq \mathcal{E}_{\mathcal{A}} \cup \mathcal{E}_{\mathcal{B}}
$$

Proof 3.

$$
\begin{aligned}
(A \otimes B)_{i j} & =\bigoplus_{k=1}^{n}\left(a_{i k} \otimes b_{k j}\right) \\
& =\cdots \oplus\left(a_{i i} \otimes b_{i j}\right) \oplus \cdots \oplus\left(a_{i j} \otimes b_{j j}\right) \oplus \cdots \\
& =\cdots \oplus b_{i j} \oplus \cdots \oplus a_{i j} \oplus \cdots
\end{aligned}
$$

Consequently, $(A \otimes B)_{i j}=\varepsilon$ is only possible if both $a_{i j}=\varepsilon$ and $b_{i j}=\varepsilon$. Hence, if either $\mathcal{G}_{A}$ or $\mathcal{G}_{B}$ has an edge $(j, i)$, this will also be an edge of $\mathcal{G}_{A \otimes B}$.

Lemma 4.3. Consider a matrix $A \in \mathbb{R}_{\max }^{n \times n}$ and the corresponding precedence graph $\mathcal{G}_{A}=(\mathcal{N}, \mathcal{E})$. Let $(i, i) \in \mathcal{E}$ $\forall i \in \mathcal{N}$, and let all edges have weight e, i.e., the diagonal elements of $A$ are all equal to e, while the off-diagonal elements are either e or $\varepsilon . \mathcal{G}_{A}$ is strongly connected if and only if $A^{k}=E \forall k \geq n-1$.

Proof 4. See Monajemi Nejad et al. (2009).

We will now investigate whether, given the set $\mathcal{A}=\left\{A_{(1)}\right.$, $\left.\cdots, A_{\left(n_{m}\right)}\right\}$ or, equivalently, the set $\left\{\mathcal{G}_{(1)}, \cdots, \mathcal{G}_{\left(n_{m}\right)}\right\}$, we can find a sequence $A_{k}, k=0,1, \cdots$, of matrices from $\mathcal{A}$ such that (10) holds.

Theorem 4.2. There exists a sequence $A_{k}, k=0,1, \cdots$, $A_{k} \in \mathcal{A}$ such that (10) holds if and only if $\bigcup_{i=1}^{n_{m}} \mathcal{G}_{(i)}$ is a strongly connected graph.

Proof 5. Sufficiency can be shown by contradiction. If there exists no sequence such that $(10)$ holds, then $\left(A_{(1)} \otimes\right.$ $\left.\cdots \otimes A_{\left(n_{m}\right)}\right)^{k} \neq E, \forall k$. Then, according to Lemma 4.3 the precedence graph of the matrix $A_{(1)} \otimes \cdots \otimes A_{\left(n_{m}\right)}$ is not a strongly connected graph. Since according to Lemma 4.2

$$
\mathcal{E}_{\left(A_{(1)} \otimes \cdots \otimes A_{\left(n_{m}\right)}\right)} \supseteq \mathcal{E}_{A_{(1)}} \cup \cdots \cup \mathcal{E}_{A_{\left(n_{m}\right)}},
$$

$\bigcup_{i=1}^{n_{m}} \mathcal{G}_{(i)}$ cannot be strongly connected.

Necessity can also be demonstrated by contradiction. Assuming that the union graph is not strongly connected, it has to be shown, that there exists no $k$ such that (10) holds. Since $\bigcup_{i=1}^{n_{m}} \mathcal{G}_{(i)}$ is not strongly connected, $\bigcup_{i=0}^{k-1} \mathcal{G}_{i} \forall k$ is not strongly connected, for all $k$ and all sequences $\mathcal{G}_{i}$, $i=0,1,2, \cdots$, where $\mathcal{G}_{i} \in\left\{\mathcal{G}_{(1)}, \cdots, \mathcal{G}_{\left(n_{m}\right)}\right\}$. As $\bigcup_{i=0}^{k-1} \mathcal{G}_{i}$ is the precedence graph of $\bigoplus_{i=0}^{k-1} A_{i}$, Lemma 4.3 implies

$$
\left(\bigoplus_{i=0}^{k-1} A_{i}\right)^{\zeta} \neq E, \forall \zeta
$$

Let us choose $\zeta=k-1$. Then, there exist $i$ and $j$ such that

$$
\begin{aligned}
\left(\left(\bigoplus_{i=0}^{k-1} A_{i}\right)_{i j}^{k-1}\right. & =\left(A_{k-1} \oplus A_{k-2} \oplus \cdots \oplus A_{0}\right)_{i j}^{k-1} \\
& =\left(A_{k-1}\right)_{i j}^{k-1} \oplus \cdots \oplus\left(A_{0}\right)_{i j}^{k-1} \oplus \cdots \\
& \oplus\left(A_{k-1} \otimes A_{k-2} \otimes \cdots \otimes A_{0}\right)_{i j}=\varepsilon .
\end{aligned}
$$

This implies $\left(A_{k-1} \otimes A_{k-2} \otimes \cdots \otimes A_{0}\right)_{i j}=\varepsilon$, i.e., $A_{k-1} \otimes$ $A_{k-2} \otimes \cdots \otimes A_{0} \neq E$, and therefore (10) does not hold. $\square$

Theorem 4.2 presents a useful criterion to determine, whether in general it is possible to achieve max-consensus for some given graphs describing a switching communication topology.

\subsection{Strong Max-Consensus in Switching Topologies for Arbitrary Switching Sequences}

In many cases the switching between communication topologies is not controllable, and a switch to another topology is caused by changes in the environment. Theorem 4.3 investigates the necessary and sufficient conditions for the communication topology graphs to achieve strong max-consensus for an arbitrary switching sequence. 
Theorem 4.3. Strong max-consensus is achieved for an arbitrary sequence $A_{k}, k=0,1, \cdots, A_{k} \in\left\{A_{(1)}, \cdots, A_{\left(n_{m}\right)}\right\}$ if and only if the precedence graphs $\mathcal{G}_{(l)}$ of $A_{(l)}, l=$ $1, \cdots, n_{m}$, are all strongly connected. Then, consensus will be achieved within at most $n-1$ communication instants. Proof 6. Necessity is obvious from Monajemi Nejad et al. (2009), as the time-invariant scenario $A_{k}=A_{(l)}, k=$ $0,1, \cdots, l \in\left\{1, \cdots, n_{m}\right\}$ is a special case.

Sufficiency is shown as follows. (9) implies:

$$
\begin{aligned}
x(n-1) & =A_{n-2} \otimes A_{n-3} \otimes \cdots \otimes A_{0} \otimes x_{0}, \\
\text { with } A_{k} & \in\left\{A_{(1)}, \cdots, A_{\left(n_{m}\right)}\right\} \forall k=\{0, \cdots, n-2\} .
\end{aligned}
$$

If

$$
A=A_{n-2} \otimes A_{n-3} \otimes \cdots \otimes A_{0}=E,
$$

max-consensus is achieved.

To show this, consider

$$
\begin{aligned}
(A)_{i j}= & \quad\left(A_{n-2} \otimes A_{n-3} \otimes \cdots \otimes A_{0}\right)_{i j} \\
= & \bigoplus_{k=1}^{n}\left(( A _ { n - 2 } ) _ { i k } \otimes \left(\bigoplus _ { m = 1 } ^ { n } \left(\left(A_{n-2}\right)_{k m} \otimes(\cdots\right.\right.\right. \\
& \otimes\left(\bigoplus_{p=1}^{n}\left(\left(A_{2}\right)_{l p} \otimes\left(\bigoplus_{q=1}^{n}\left(\left(A_{1}\right)_{p q} \otimes\left(A_{0}\right)_{q j}\right)\right)\right)\right) .
\end{aligned}
$$

Now we have to show that $(A)_{i j}=\mathrm{e} \forall i, j$, i.e., all multiplicative terms in the equation above are equal to e.

$\forall j \exists q$ such that $\left(A_{0}\right)_{q j} \neq \varepsilon$, since according to Lemma 4.1 in $\mathcal{G}_{0}^{\prime}=\left(\{j\}, \mathcal{E}_{0}^{\prime}\right) \subset \mathcal{G}_{0}$ there exists at least one node $q \neq j$ such that $\left(A_{0}\right)_{q j}=e$.

According to Lemma 4.1 in $\mathcal{G}_{1}^{\prime}=\left(\{j, q\}, \mathcal{E}_{1}^{\prime}\right) \subset \mathcal{G}_{1}$ there exists at least one node $p \neq q \neq j$ such that either $\left(A_{1}\right)_{p q}=e$ or $\left(A_{1}\right)_{p j}=e$. Then, recalling the special structure of the matrices $A_{i},\left(\left(A_{1}\right) \otimes\left(A_{0}\right)\right)_{p j}=\mathrm{e}$.

After $n-1$ instants of communication, according to Lemma 4.1 and because of strong connectivity of $\mathcal{G}_{n-2}$, considering $\mathcal{G}_{n-2}^{\prime}=\left(\{\underbrace{j, q, \cdots, k}_{n-1 \text { nodes }}\}, \mathcal{E}_{n-2}^{\prime}\right) \subset \mathcal{G}_{n-2}$ there exists at least one edge from the only remaining node in $\mathcal{N} \backslash$ $\{j, q, p, \cdots, k\}$ to a node $\{j, q, p, \cdots, k\}$, i.e. $\left(A_{n-2} \otimes A_{n-3} \otimes\right.$ $\left.\cdots \otimes A_{0}\right)_{i j}=\mathrm{e} \forall i, j \in \mathcal{N}$.

The following statement about the required number of communication instants can be shown using similar arguments.

Consider the set $\mathcal{G}_{(1)}, \cdots, \mathcal{G}_{\left(n_{m}\right)}$, where all $\mathcal{G}_{(i)}$ are strongly connected. From Monajemi Nejad et al. (2009), we know that for the time-invariant case, $\mathcal{G}_{k}=\mathcal{G}_{(l)}$, the required number of communication instants, $m_{l}$, is the maximum of the shortest path lengths between any pair of nodes in $\mathcal{G}_{(l)}$. In the switching topology case, the required number of communication instants, $m$ is

$$
\min _{l=1, \cdots, m_{n}}\left(m_{l}\right) \leq m \leq \max _{l=1, \cdots, m_{n}}\left(m_{l}\right) .
$$

In summary, we have shown that strong max-consensus is achieved for an arbitrary switching sequence if and only if each graph is strongly connected. Furthermore, the required number of communication instants is bounded by (12), and is $n-1$, in the worst case.

\section{WEAK MAX-CONSENSUS IN SWITCHING TOPOLOGIES}

We now consider the case of weak max-consensus as defined in Section 3. Not surprisingly, it will turn out that conditions are weaker for this case.

\subsection{Weak Max-Consensus in Switching Topologies for Specific Switching Sequences}

Theorem 5.1. Let $A_{k}, k=0,1,2, \cdots$, be a sequence of matrices with $A_{k}=A_{\left(l_{k}\right)}$. Denote the maximum of all initial information states by $\hat{x}_{0}$, i.e.,

$$
\hat{x}_{0}:=\max _{i=1, \cdots, n}\left\{x_{i}(0)\right\}
$$

and partition the index set $\mathcal{N}=\{1, \cdots, n\}$ by:

$$
\begin{aligned}
& \mathcal{N}_{1}:=\left\{i \mid x_{i}(0)=\hat{x}_{0}\right\}, \\
& \mathcal{N}_{2}:=\mathcal{N} \backslash \mathcal{N}_{1} .
\end{aligned}
$$

Then, a necessary and sufficient condition for maxconsensus is that:

$$
(\exists k \in \mathbb{N}) \quad\left(\forall i \in \mathcal{N}_{2}\right) \quad\left(\exists m \in \mathcal{N}_{1}\right):(A)_{i m}=\mathrm{e},
$$

where $A:=A_{\left(l_{k-1}\right)} \otimes \cdots \otimes A_{l_{0}}$.

Proof 7. To show sufficiency, recall that according to Lemma 4.2, (13) implies

$$
\left(A_{l_{q-1}} \otimes \cdots \otimes A\right)_{i m}=\mathrm{e}, \forall q>k .
$$

We then have $\forall i \in \mathcal{N}_{2}, \forall q>k$

$$
\begin{aligned}
x_{i}(q)= & \bigoplus_{j \in \mathcal{N}_{1}}\left(A_{l_{q-1}} \otimes \cdots \otimes A\right)_{i j} \otimes \underbrace{x_{j}(0)}_{\hat{x}_{0}} \\
& \bigoplus_{j \in \mathcal{N}_{2}}\left(A_{l_{q-1}} \otimes \cdots \otimes A\right)_{i j} \otimes x_{j}(0) \\
= & \hat{x}_{0} .
\end{aligned}
$$

We also trivially have

$$
x_{i}(q)=\hat{x}_{0} \quad \forall i \in \mathcal{N}_{1}, \forall q \geq 0 .
$$

To show necessity, assume that (13) does not hold, i.e.,

$$
(\forall k \in \mathbb{N}) \quad\left(\exists i \in \mathcal{N}_{2}\right) \quad\left(\forall m \in \mathcal{N}_{1}\right):(A)_{i m}=\varepsilon .
$$

This implies that there exists a node $i$ in $\mathcal{N}_{2}$ such that, $\forall k, x_{i}(k)$ does not depend on $\hat{x}_{0}$, hence consensus is not achieved.

Theorem 5.2 investigates whether, given the set $\mathcal{A}=\left\{A_{(1)}\right.$, $\left.\cdots, A_{\left(n_{m}\right)}\right\}$, or, equivalently, the set $\left\{\mathcal{G}_{(1)}, \cdots, \mathcal{G}_{\left(n_{m}\right)}\right\}$, we can find a sequence $A_{k}, k=0,1, \cdots$, of matrices from $\mathcal{A}$ such that (13) holds.

Theorem 5.2. There exists a sequence $A_{k}, k=0,1, \cdots$, $A_{k} \in \mathcal{A}$ such that (13) holds if and only if there exist subgraphs $\mathcal{G}_{T_{i}}:=\left(\mathcal{N}_{T_{i}}, \mathcal{E}_{T_{i}}\right)$, of the union graph $\bigcup_{i=1}^{n_{m}} \mathcal{G}_{(i)}$, such that the $G_{T_{i}}$ are rooted directed trees with roots in $\mathcal{N}_{1}$ and $\cup_{i} \mathcal{N}_{T_{i}} \supseteq \mathcal{N}_{2}$. 
Proof 8. The proof is omitted for the sake of brevity, but can be developed similarly to the proof of Theorem 4.2 and is based on the results from Monajemi Nejad et al. (2009).

Example 5.1. Consider the graphs in Figure 1. Let us

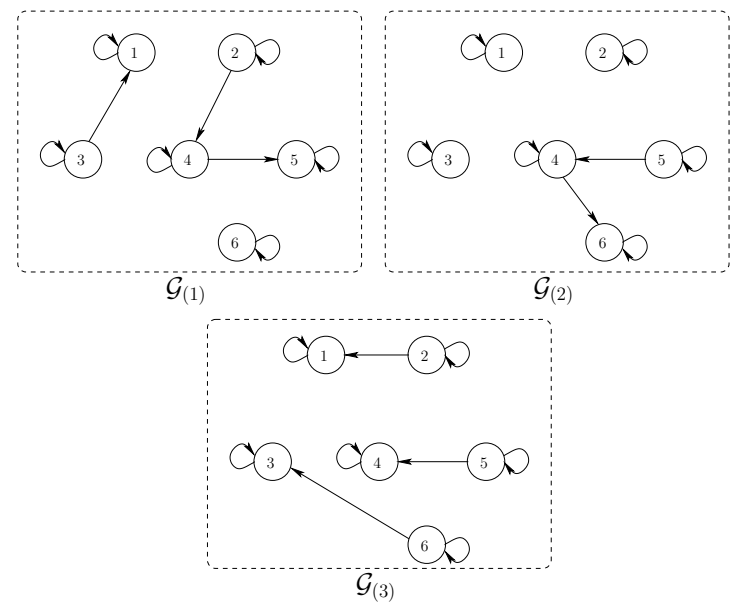

Figure 1. Communication graphs for Example 5.1.

assume that $\mathcal{N}_{1}=\{1,2\}$, i.e., $x_{1}(0)=x_{2}(0)=\hat{x}_{0}$. The condition from Theorem 5.2 is that the union graph of these three graphs (Figure 2) contains rooted directed trees $\mathcal{G}_{T_{i}}:=\left(\mathcal{N}_{T_{i}}, \mathcal{E}_{T_{i}}\right)$ with roots in $\mathcal{N}_{1}$ and $\cup_{i} \mathcal{N}_{T_{i}} \supseteq \mathcal{N}_{2}$. Obviously, in the union graph (Figure 2) there exists such a

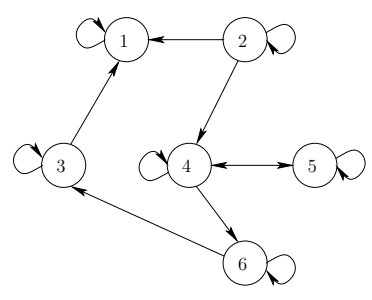

$\mathcal{G} \cup$

Figure 2. Union graph for Example 5.1.

tree with node 2 as the root and paths to all other nodes in $\mathcal{N}_{2}$ (Figure 3). Hence, a suitable sequence $\mathcal{G}_{\left(l_{i}\right)}, i=0,1, \cdots$,

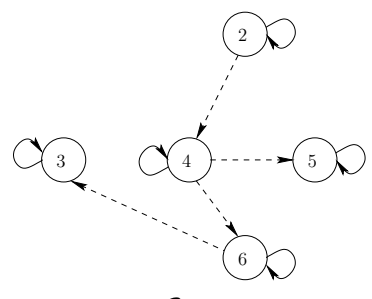

$\mathcal{G} \cup$

Figure 3. Rooted directed tree in the union graph of Figure 2.

or, equivalently, $A_{\left(l_{i}\right)}, i=0,1, \cdots$ exists. Consider, e.g. $A_{k}=A_{(1)}$ for $k=0,3,6, \cdots, A_{k}=A_{(2)}$ for $k=1,4,7, \cdots$, and $A_{k}=A_{(3)}$ for $k=2,5,8, \cdots$. Then,
$x(9)=$
$A_{8} \otimes \cdots \otimes A_{2} \otimes A_{0} \otimes x_{0}$

$$
=\left(\begin{array}{c}
x_{1}(0) \oplus x_{2}(0) \oplus x_{3}(0) \oplus x_{4}(0) \oplus x_{5}(0) \oplus x_{6}(0) \\
x_{2}(0) \\
x_{2}(0) \oplus x_{3}(0) \oplus x_{4}(0) \oplus x_{5}(0) \oplus x_{6}(0) \\
x_{2}(0) \oplus x_{4}(0) \oplus x_{5}(0) \\
x_{2}(0) \oplus x_{4}(0) \oplus x_{5}(0) \\
x_{2}(0) \oplus x_{4}(0) \oplus x_{5}(0) \oplus x_{6}(0)
\end{array}\right) .
$$

5.2 Weak Max-Consensus in Switching Topologies for Arbitrary Switching Sequences

Theorem 5.3 investigates now these necessary and sufficient conditions for achieving weak max-consensus for arbitrary switching sequences.

Theorem 5.3. Weak max-consensus is achieved for any sequence $A_{k}, k=0,1, \cdots, A_{k} \in\left\{A_{(1)}, \cdots, A_{\left(n_{m}\right)}\right\}$ if and only if there exist subgraphs $\mathcal{G}_{T_{i}}:=\left(\mathcal{N}_{T_{i}}, \mathcal{E}_{T_{i}}\right)$, of each graph $\mathcal{G}_{(i)} i \in\left\{1, \cdots, n_{m}\right\}$, such that the $G_{T_{i}}$ are rooted directed trees with roots in $\mathcal{N}_{1}$ and $\cup_{i} \mathcal{N}_{T_{i}} \supseteq \mathcal{N}_{2}$.

The maximum number of required communication instants to achieve consensus is given by:

$$
\max _{l: A_{(l)} \in A_{k}, k=0,1, \cdots}\left(\max _{i \in \mathcal{N}_{2}}\left(\min _{m \in \mathcal{N}_{1}}\left\{|m, i|_{\mathcal{G}_{(l)}, \min }\right\}\right)\right),
$$

where $|m, i|_{\mathcal{G}_{(l)} \text {, min }}$ denotes the length of the shortest path from node $m$ to node $i$ in graph $\mathcal{G}_{(l)}$.

Proof 9. Omitted for the sake of brevity, but can be developed in the same way as the proof of Theorem 4.3, using the results given for the case of fixed communication topologies (see Monajemi Nejad et al. (2009)).

Example 5.2. Consider the graphs in Figure 4. Assume

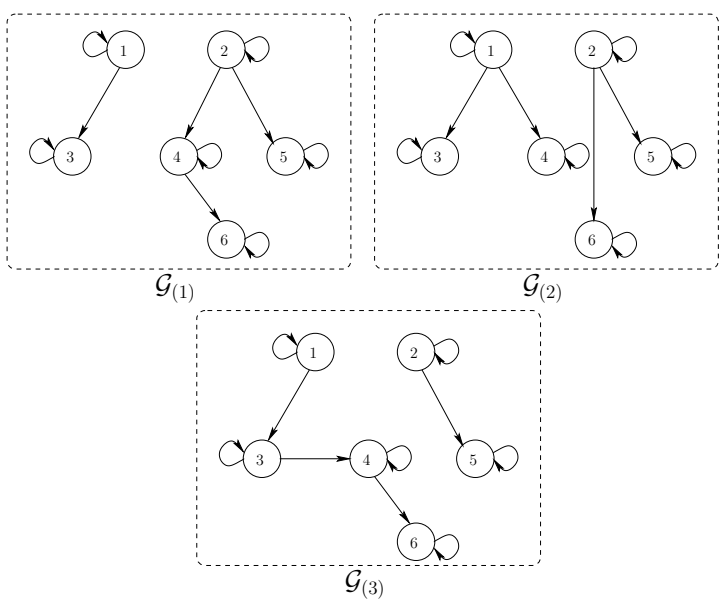

Figure 4. Communication graphs for Example 5.2

that $x_{1}(0)=x_{2}(0)=\hat{x}_{0}$, i.e., $\mathcal{N}_{1}=\{1,2\}$. The necessary and sufficient condition given in Theorem 5.3 holds. Thus, a max-consensus will be achieved, and the maximal number of communication instants is

$$
\max _{l: A_{(l)} \in A_{k}, k=0,1, \cdots}\left(\max _{i \in \mathcal{N}_{2}}\left(\min _{m \in \mathcal{N}_{1}}\left\{|m, i|_{\mathcal{G}_{(l)}, \min }\right\}\right)\right)=3 .
$$

Consider, e.g., a sequence $A_{k}, k=0,1, \cdots$, defined as follows:

$A_{k}=A_{(1)}$ for $k=0,2, \cdots$, and $A_{k}=A_{(2)}$ for $k=1,3, \cdots$. Then, we get in max-plus algebra 


$$
\left.\begin{array}{rl}
x(2)= & A_{2} \otimes A_{1} \otimes x_{0} \\
x_{1}(0) \\
x_{2}(0) \\
x_{1}(0) \oplus x_{3}(0) \\
x_{1}(0) \oplus x_{2}(0) \oplus x_{4}(0) \\
x_{2}(0) \oplus x_{5}(0) \\
x_{2}(0) \oplus x_{4}(0) \oplus x_{6}(0)
\end{array}\right)=\left(\begin{array}{c}
\hat{x}_{0} \\
\hat{x}_{0} \\
\hat{x}_{0} \\
\hat{x}_{0} \\
\hat{x}_{0} \\
\hat{x}_{0}
\end{array}\right) .
$$

Clearly it means that under the given assumption the state of a switched max-plus system consisting of $A_{1}$ and $A_{2}$ converges to $\hat{x}_{0}$, i.e., consensus is achieved after two communication instants.

According to Theorem 5.3 the maximum required instances of communication is in the worst case 3 . In maxplus algebra one can easily check, that

$$
x(3)=A_{3} \otimes A_{1} \otimes A_{3} \otimes x_{0}=\hat{X}_{0} .
$$

Similar to the case of strong max-consensus, the following statement about the required number of communication instants can be shown.

Consider the set $\mathcal{G}_{(1)}, \cdots, \mathcal{G}_{\left(n_{m}\right)}$, where the conditions in Theorem 5.3 hold for all $\mathcal{G}_{(i)}$. From Monajemi Nejad et al. (2009), we know that for the time-invariant case, $\mathcal{G}_{k}=\mathcal{G}_{(l)}$, the required number of communication instants, $m_{l}$, is given by

$$
\max _{i \in \mathcal{N}_{2}}\left(\min _{j \in \mathcal{N}_{1}}\left\{|j, i|_{\mathcal{G}_{(l)}, \min }\right\}\right) .
$$

In the switching topology case, the required number of communication instants, $m$ is

$$
\min _{l=1, \cdots, m_{n}}\left(m_{l}\right) \leq m \leq \max _{l=1, \cdots, m_{n}}\left(m_{l}\right)
$$

In summary we have shown under which conditions weak max-consensus is achieved. Obviously, the conditions are more restricted if the switching sequence is arbitrary (Theorem 5.3). On the other hand, being able to choose freely a switching sequence relaxes the conditions. Theorem 5.2 give a useful criterion for the existence of a feasible switching sequence, such that max-consensus is achievable.

\section{EXAMPLES AND SIMULATION RESULTS}

In the sequel some simulation results are given for both strong and weak max-consensus. As a motivating example we consider the following scenario: a network of four agent is supposed to meet at a minimum time at a particular place. The agents should agree on the minimal feasible time via communication at discrete instants of time. The following simulations demonstrate under which conditions on communication topology graphs max-consensus is achieved.

\subsection{Strong Max-Consensus:}

Assume that the set of feasible communication graphs is given by $\left\{\mathcal{G}_{(1)}, \mathcal{G}_{(2)}, \mathcal{G}_{(3)}\right\}$ (Figure 5). The sequence of communication topology is given by $\mathcal{G}_{k} \in\left\{\mathcal{G}_{(1)}, \mathcal{G}_{(2)}, \mathcal{G}_{(3)}\right\}$, $k=0,1, \cdots$. Obviously, $\bigcup_{i=1}^{3} \mathcal{G}_{(i)}$ is strongly connected. Hence, a suitable sequence, e.g., $A_{k}=A_{(1)}$ for $k=0,3, \cdots$,
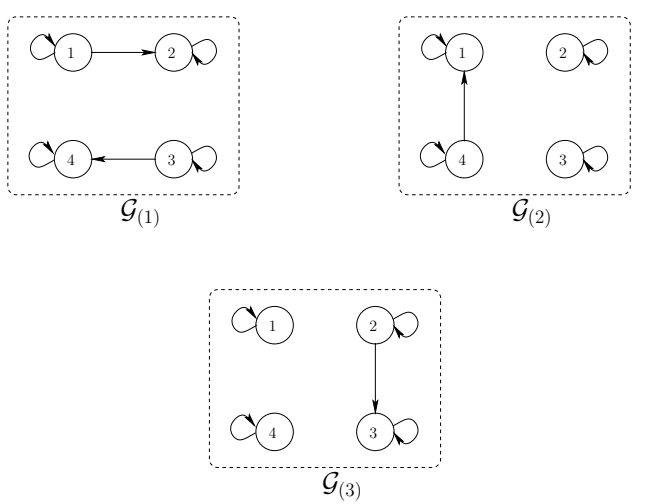

Figure 5. Communication graphs for simulation in Section 6.1.

$A_{k}=A_{(2)}$ for $k=1,4, \cdots$, and $A_{k}=A_{(1)}$ for $k=2,5, \cdots$ max-consensus can be achieved.

$$
=A_{0} \otimes x_{0}=\left(\begin{array}{c}
x_{1}(0) \\
x_{1}(0) \oplus x_{2}(0) \\
x_{3}(0) \\
x_{3}(0) \oplus x_{4}(0)
\end{array}\right)
$$

$x(3)=A_{2} \otimes A_{1} \otimes A_{0} \otimes x_{0}=\left(\begin{array}{c}x_{1}(0) \oplus x_{3}(0) \oplus x_{4}(0) \\ x_{1}(0) \oplus x_{2}(0) \\ x_{1}(0) \oplus x_{2}(0) \oplus x_{3}(0) \\ x_{3}(0) \oplus x_{4}(0)\end{array}\right)$

$x(k)=E \otimes x_{0}=\left(\begin{array}{l}x_{1}(0) \oplus \cdots \oplus x_{4}(0) \\ x_{1}(0) \oplus \cdots \oplus x_{4}(0) \\ x_{1}(0) \oplus \cdots \oplus x_{4}(0) \\ x_{1}(0) \oplus \cdots \oplus x_{4}(0)\end{array}\right), \forall k \geq 6$.

In Figure 6 simulation results are given for an initial vectors of information state $x_{o}=(1,2,3,4)^{T}$. For this vector, as for every other vector $x_{0} \in \mathbb{R}^{n}$, max-consensus is achieved after maximal 6 communication instants.

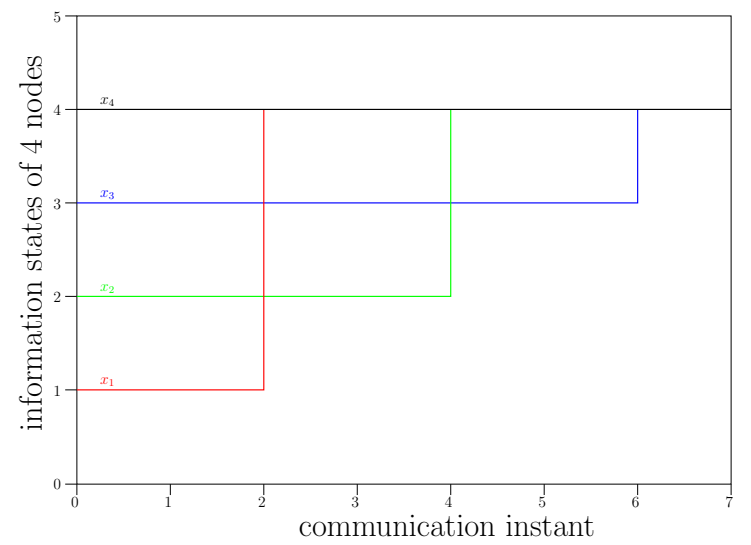

Figure 6. Simulation results for $x_{0}$

\subsection{Weak Max-Consensus:}

Assume that the feasible topologies are given by the graphs in Figure 1 from example 5.1. The condition in Theorem 5.2 holds if $\mathcal{N}_{1}=\{2\}$. In the following, the simulation results for two initial vectors of information states are depicted in Figure 7 and Figure 8. 
Figure 7 presents the simulation results for $x_{0}=(1,2,3,4,5$, $6)^{T}$, where the necessary and sufficient conditions of Theorem 5.2 are violated. Obviously, the information states of the agents do not converge to the maximum value.

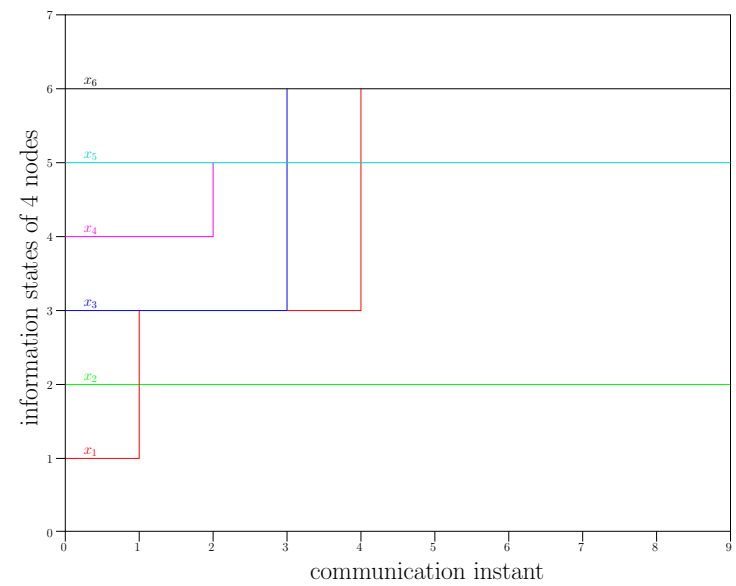

Figure 7. Simulation results for $x_{0}$

For an initial vector $x_{0}^{\prime}=(2,6,3,1,4,5)^{T}$ max-consensus is achieved as illustrated in Figure 8.

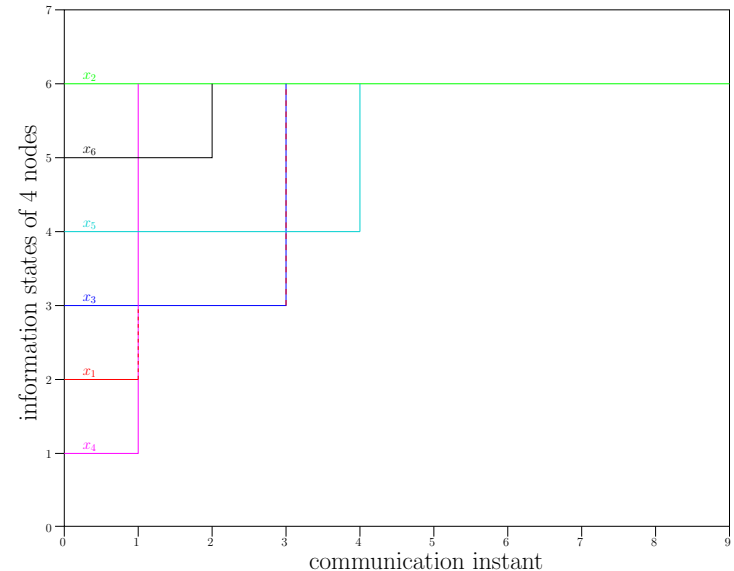

Figure 8. Simulation results for $x_{0}^{\prime}$

\section{CONCLUSIONS}

A main ingredient in any distributed cooperative control system is an efficient consensus mechanism. In this paper, we have investigated max-consensus problems in switching communication topologies, where one aims at determining the maximal value of all agents' initial information states through time-variant local communication.

We have proposed to use max-plus algebra to analyze max-consensus algorithms. In this framework, we obtain a switched linear system representation of the investigated consensus algorithm. We have studied convergence conditions for both strong and weak max-consensus for time-variant communication topologies. Note that this approach can also be used to study min-consensus problems - one then seeks the maximum of $-x_{0}$.

\section{REFERENCES}

Baccelli, F., Cohen, G., Olsder, G.J., and Quadrat, J.P. (2001). Synchronization and Linearity - An Algebra for Discrete Event Systems. Wiley, web edition.

Cao, M., Morse, A.S., and Anderson, B.D.O. (2008). Reaching a consensus in a dynamically changing environment: Convergence rates, measurement delays, and asynchronous events. SIAM J. Control Optim., 47(2), 601-623.

Cortés, J. (2008). Distributed algorithms for reaching consensus on general functions. Automatica, 44(3), 726737.

De Schutter, B. and van den Boom, T. (2008). Max-plus algebra and max-plus linear discrete event systems: An introduction. In Proceedings of the 9th International Workshop on Discrete Event Systems (WODES'08), 3642. Göteborg, Sweden.

Diestel, R. (2005). Graph Theory, volume 173 of Graduate Texts in Mathematics. Springer-Verlag, Heidelberg, third edition.

Feddema, J., Lewis, C., and Schoenwald, D. (2002). Decentralized control of cooperative robotic vehicles: theory and application. Robotics and Automation, IEEE Transactions on, 18(5), 852-864.

Gil, A., Passino, K., and Cruz, J. (2008). Stable cooperative surveillance with information flow constraints. IEEE Transactions on Control Systems Technology,, 16(5), 856-868.

Heidergott, B., Olsder, G., and van der Woude, J. (2005). Max Plus at Work: Modeling and Analysis of Synchronized Systems: A Course on Max-Plus Algebra and Its Applications (Princeton Series in Applied Mathematics). Princeton University Press.

Lynch, N.A. (1996). Distributed Algorithms. Morgan Kaufmann Publishers Inc., San Francisco, CA, USA.

Monajemi Nejad, B., Attia, S., and Raisch, J. (2009). Maxconsensus in a max-plus algebraic setting: The case of fixed communication topologies. In XXII International Symposium on Information, Communication and Automation Technologies (ICAT) 2009.

Moreau, L. (2005). Stability of Multiagent Systems With Time-Dependent Communication Links. IEEE Trans. on Automatic Control, 50(2), 169-182.

Olfati-Saber, R. and Murray, R. (2004). Consensus problems in networks of agents with switching topology and time-delays. IEEE Transactions on Automatic Control,, 49(9), 1520 - 1533.

Ren, W. and Beard, R.W. (2008). Distributed Consensus in Multi-vehicle Cooperative Control. Communications and Control Engineering. Springer-Verlag, London.

Roy, S., Saberi, A., and Herlugson, K. (2005). A controltheoretic perspective on the design of distributed agreement protocols. Proceedings of the American Control Conference, 2005., 1672-1679 vol. 3.

Tahbaz-Salehi, A. and Jadbabaie, A. (2006). A oneparameter family of distributed consensus algorithms with boundary: From shortest paths to mean hitting times. 45th IEEE Conference on Decision and Control, 2006., 4664-4669.

Wang, P., Yee, J., Xia, C., Mokuno, M., and Hadaegh, F. (2004). Cooperative control of a magnetically levitated interferometer. In Proceedings of the IEEE International Conference on Control Applications, 2004., 18-25 Vol.1. 